\title{
Functional scale-free networks in the two-dimensional Abelian sandpile model
}

\author{
M. Zarepour, ${ }^{1}$ M. D. Niry, ${ }^{1,2, *}$ and A. Valizadeh ${ }^{1,3}$ \\ ${ }^{1}$ Department of Physics, Institute for Advanced Studies in Basic Sciences (IASBS), Zanjan 45137-66731, Iran \\ ${ }^{2}$ Center for Research in Climate Change and Global Warming (CRCC), \\ Institute for Advanced Studies in Basic Sciences (IASBS), Zanjan 45137-66731, Iran \\ ${ }^{3}$ School of Cognitive Sciences, Institute for Research in Fundamental Sciences (IPM), Tehran - Iran
}

(Dated: June 29, 2015)

\begin{abstract}
Recently, similarity of the functional network of the brain and the Ising model was investigated in Nat. Phys. 6, 744 (2010). This similarity supports the idea that the brain is a self-organized critical system. In this study we derive functional network of the $2 \mathrm{D}$ BTW sandpile model as a self-organized critical model, and compare its charachteristics with those of the functional network of the brain, obtained from functional magnetic resonance imaging (fMRI).
\end{abstract}

PACS numbers: $\quad$ 05.65.+b,87.18.-h,87.19.1f,89.75.Da

Keywords: Self-organized systems,Biological complexity,MRI,Systems obeying scaling laws

\section{INTRODUCTION}

Brain consists of Tens of Billions of highly nonlinear components and exhibits collective dynamics which in many aspects resemble some of the well-known phenomena in statistical physics [1]. Neurons can be considered as leaky integrators with a highly nonlinear inputoutput transfer function [2]. The individual neurons integrate synaptic inputs received from other neurons and if a threshold is crossed, they send their activity back to the network. The neurons receiving stimulation can in turn exceed threshold and transfer the activity to other neurons and initiate an avalanche whose spatial and temporal length determines how much the perturbation can survive in the network. This process can be seen in many other complex systems in which individual units with a threshold, integrate and then send energy back to the system [3-6]. In such systems the perturbations can die out in very short distances or propagate through the network as avalanches with no characteristic time and length scale, indicating the system is in critical state [7]. Unlike the conventional critical states in equilibrium statistical mechanics which occur with fine-tuned parameters, these critical states are attractors reached by starting far from equilibrium; they are insensitive to parameters and many dynamical systems with extended spatial degrees of freedom evolve into such self-organized critical states [7].

With no dominant characteristic scale the process show scale-free behavior characterized by power law distribution. Power law scaling behavior in such systems reflects the tendency of complex systems to develop correlation that decays more slowly and extend over larger distances in time and space than the mechanisms of the underlying process would suggest [8-10]. Such power law statistics in neuronal activity has been reported in several experimental and modeling studies (see e.g. [1, 11-20], and as an

\footnotetext{
* Author to whom correspondence should be addressed. Electronic address: m.d.niry@iasbs.ac.ir; URL: http://www.iasbs.ac. $\mathrm{ir} / \sim \mathrm{m} . \mathrm{d}$. niry/
}

opposite view see [21]). Recent works on healthy brain at small and large scales show that the brain dynamics is not composed of completely random patterns or of periodic oscillations [22], but in the critical state characterized by a scale-free behavior. Studies show that several aspects of information processing by neural networks, including dynamic range of processing the stimulus and the amount of information which can be transferred, are optimized in the critical state [11, 23-25].

Scaling behavior can be seen in the functional connectivity of the healthy brain [26-28] and possible deficit in functional connectivity among brain regions contribute to the cognitive dysfunction such as schizophrenia [29], Alzheimer and epilepsy [28, 30, 31]. Functional connectivity is a measure of correlation among voxels signal in the functional magnetic resonance imaging (fMRI) from the brain regions. Although in healthy adult brains structural and functional connectivity show positive correlation, functional connectivity can be observed between the regions with no direct structural connection [32]. Interestingly functional connectivity has been shown to be robust against structural deficits. Experiments show that there are compensatory reorganization mechanisms which retain the functional coordination between brain regions after removing structural connections [33, 34].

The data associated with fMRI for a brain in a resting state (a condition in which the brain is not performing an explicit task) indicate that the characteristic properties of the human brain's functional network have striking similarities with properties extracted from the dynamics of the 2-dimensional Ising model at its critical state [1, $14,35,36]$. With each spin considered as a vertex of a graph in the Ising model, spins that are more correlated are connected to each other with undirected edges and in this manner functional network of the Ising model is constructed. It is shown that at the critical temperature the functional network of the Ising model would be scalefree with power law degree distribution, similar to that of the functional network of the brain $[1,13,27]$.

Small-world [37], scale-free [38] functional network of the brain at the rest state is a strong evidence of the 
self-organized criticality (SOC) in the brain [1]. This behavior encourages us to using the BTW sandpile model, suggested by Bak, Tang and Wiesenfeld, instead of the 2-dimensional Ising model to compare the results with the behavior of the brain functional network [7, 39, 40]. BTW model represents the simple but complex nonequilibrium dynamics of a driven dissipative system with simple short-range interaction of the components, keeping pressure on its cells until they exceed the specific threshold (non-linearity in the model) and progress toward critical state and leads it to produce the events have been called avalanches [10]. That is the first and the simplest example of self-organizing critical systems [39].

In present study we extract the functional network of the sandpile model with similar method introduced in $[14,41]$. We preferred the sandpile model to the Ising model for two significant reasons. First, BTW model can be investigated as a cellular automata [42], where evolution rule of each cell is speculatively similar to the evolution rule of an integrate-and-fire neuron [43]. So, one can expect scaling behavior of 2-dimensional BTW model to be similar to the scaling behavior of integrateand-fire neurons on a regular square lattice. Second, unlike the Ising model, where emerging power law behavior (criticality), is only possible by controlling temperature of the model around the critical point, BTW model shows critical behavior in a self-organized manner, without any tunable external parameter $[2,43,44]$.

\section{THE ABELIAN SANDPILE MODEL}

Self-organized criticality is introduced in 1987, based on the dynamics of complex systems with a critical attractor in their phase space portrait [7, 39]. Many of the complex phenomena in the nature which show scaling behavior [10] and make pink noise [45-47] -two specific signs of SOC- can be explained with the same concepts [10]. The BTW sandpile model is introduced as the most basic system that provides all of main features of SOC systems $[7,39,44]$.

The BTW sandpile model in the two-dimensional $L \times L$ square lattice is an Abelian sandpile model [48]. The lattice contains $L^{2}$ sites and each lattice site $(i, j)$ has an associated variable $h_{i j}$, where $h_{i j}=1,2,3,4$ shows the height of the site [49]. In each step, a random site $(i, j)$ is chosen, and its height is increased by one:

$$
h_{i, j} \longrightarrow h_{i, j}+1 \text {. }
$$

If the height of the site exceeds the threshold, $h_{c}=4$, a toppling occurs. The toppling is characterized by the following rules [39, 48]:

$$
\begin{cases}h_{i, j} & \longrightarrow h_{i, j}-h_{c} \\ h_{i, j \pm 1} \longrightarrow h_{i, j \pm 1}+1 \\ h_{i \pm 1, j} \longrightarrow h_{i \pm 1, j}+1\end{cases}
$$

This may in turn lead to other unstable sites; so the update rule Eq. (2) is continued for such sites till stable state is reached, where the height of all the sites is below the threshold, $h_{c}$. In this model the system boundary is open, so for a toppling event which occurs at the boundary of the lattice, grains that fall outside the lattice are disposed. The final state of the system obtained at each step will be used at the next step by selecting another random site. The simulation begins from random initial condition, which may be a transient state of the system. Dynamics of the sandpile model starts from such transient state and eventually reaches a steady state which is suitable for calculating statistical quantities. According to our knowledge when the dynamics of BTW model reaches to its steady state, it will not produce transient state in the next steps [44]. The well-known Burning algorithm [44] is used to distinguish the transition from transient state to the steady state, and thereafter we perform all the next steps to calculate the statistical quantities.

We use the two-point correlation coefficient between height of arbitrary sites $\mathbf{x}=(i, j)$ and $\mathbf{x}^{\prime}=\left(i^{\prime}, j^{\prime}\right)$

$$
r\left(h_{\mathbf{x}}, h_{\mathbf{x}^{\prime}}\right)=\frac{\left\langle h_{\mathbf{x}}(t) h_{\mathbf{x}^{\prime}}(t)\right\rangle-\left\langle h_{\mathbf{x}}(t)\right\rangle\left\langle h_{\mathbf{x}^{\prime}}(t)\right\rangle}{\sigma\left(h_{\mathbf{x}}\right) \sigma\left(h_{\mathbf{x}^{\prime}}\right)},
$$

to measure the degree of linear dependency between any pair of sites [35]. In Eq. (3) $\sigma^{2}\left(h_{\mathbf{x}}\right)=\left\langle h_{\mathbf{x}}^{2}\right\rangle-\left\langle h_{\mathbf{x}}\right\rangle^{2}$ and $\langle\cdots\rangle$ represents the average taken over the length of the time series.

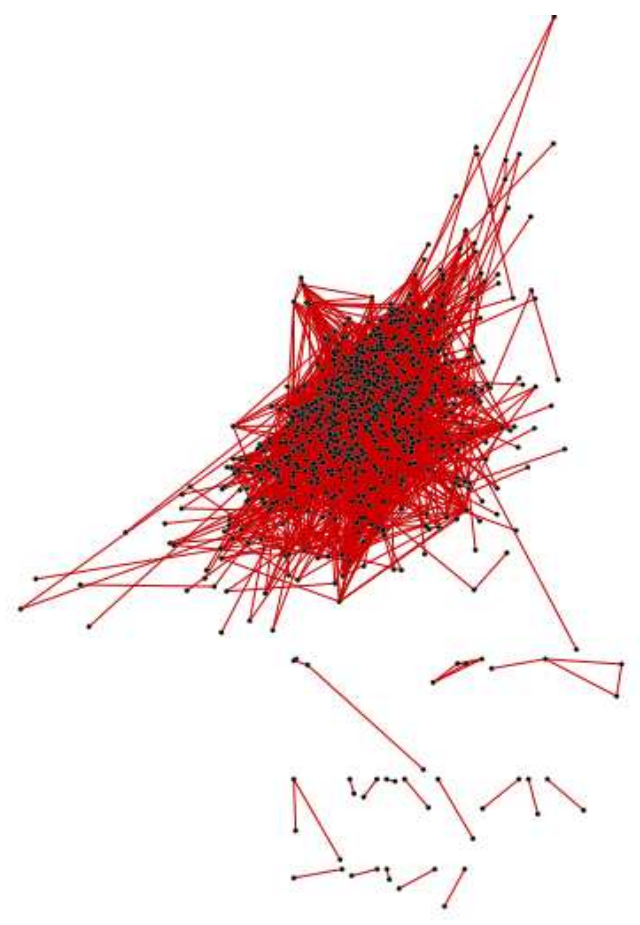

FIG. 1. (Color online) Functional network of the twodimensional BTW sandpile model for lattice size $L=32$.

If the correlation coefficient $r$, is higher than a threshold $r_{c}$, we assume that the two sites are functionally con- 
nected. Each site in the two-dimensional lattice of the sandpile model is considered as a vertex of a complex network. Any two vertices corresponding to the two arbitrary sites are connected to each other with an edge if the correlation coefficient $r$ between them is higher than $r_{c}$. Correlation matrix of the sandpile model was used to produce the adjacency matrix of the network. This network is called the functional network of model. Finally, network visualization (Fig. 1) and analysis were performed by using the software Cytoscape 3.0.2 [50], also part of analysis reported in Fig. 2 is performed by SNAP 2.4 [51].

\section{A SUITABLE CHOICE FOR $r_{c}$}

In this section we argue how we choose a suitable threshold for the correlation coefficient $r_{c}$, over which the nodes are assumed to be functionally connected. We have calculated the characteristic parameters for the functional network of the sandpile model with lattice size $L=64$ for different values of $r_{c}$, and for an equivalent random network with the same number of nodes and links. The results are shown in Fig. 2. As is seen in Fig. 2(a), for $r_{c} \lesssim 0.8$ the network has a large clustering coefficient which is a signature of the regular and small-world networks. For $r_{c}>0.6$, the ratio of the clustering coefficient of the functional network to the same parameter calculated for the equivalent random network $c / c_{\text {ran }} \gg 1$. For threshold values less than 0.4 , the clustering coefficients of the random network and the functional network get closer together since both the networks turn into all-to-all connected network.

In Fig. 2(b) we have shown the normalized size of the largest connected network $n_{c} / N$ versus the threshold value for correlation coefficient $r_{c}$, where $n_{c}$ and $N$ are the number of vertices in the largest connected graph and the total number of vertices in the network, respectively. $n_{c} / N \rightarrow 1$ means all of the nodes in the network are members of the largest connected graph and $n_{c} / N \ll 1$ means that largest connected graph is too small. The total possible number of edges in a fully connected graph of the size $n_{c}$ is $n_{c}\left(n_{c}-1\right) / 2$. We can define the density of links $\rho_{l}$ as the ratio of the number of edges, $n_{l}$, to the total possible number of edges in the largest connected graph, $2 n_{l} /\left[n_{c}\left(n_{c}-1\right)\right]$. Empty squares in Fig. 2(b) show $\rho_{l}$ : In the range of 0.5 to 0.8 the number of nodes in the graph has an acceptable value and graph is sparse. For $r_{c} \lesssim 0.5$, the number of links in the functional network is too large and the network approaches to a fully connected network. Above this range $r_{c} \gtrsim 0.8$ the number of nodes is too small and the statistical average is not reliable. The density of links $\rho_{l}$ in Fig. 2(b) is calculated by averaging over 3 sets of data. In all data points with $r_{c}<0.9$ error bars are smaller than symbol size, but for $r_{c} \geq 0.9$ error is increased and is of the order of 0.01 .

For small values of $r_{c}$, the diameter $d$ and average shortest path length $l$ are small similar to the ran-

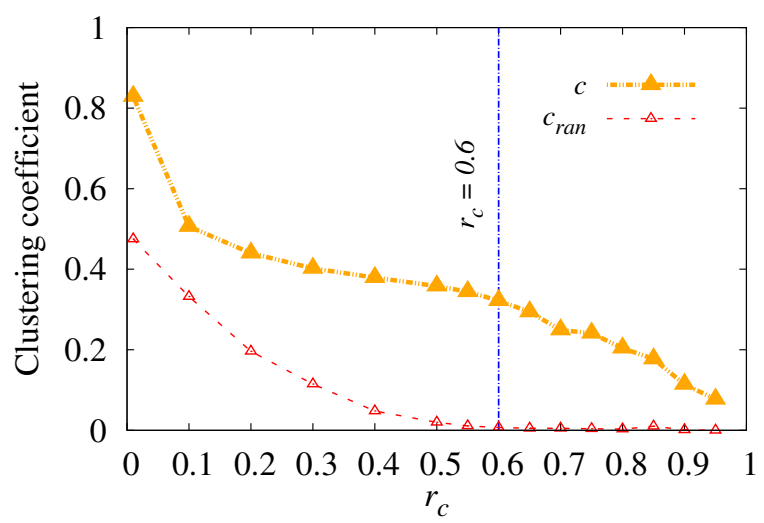

(a)



(b)

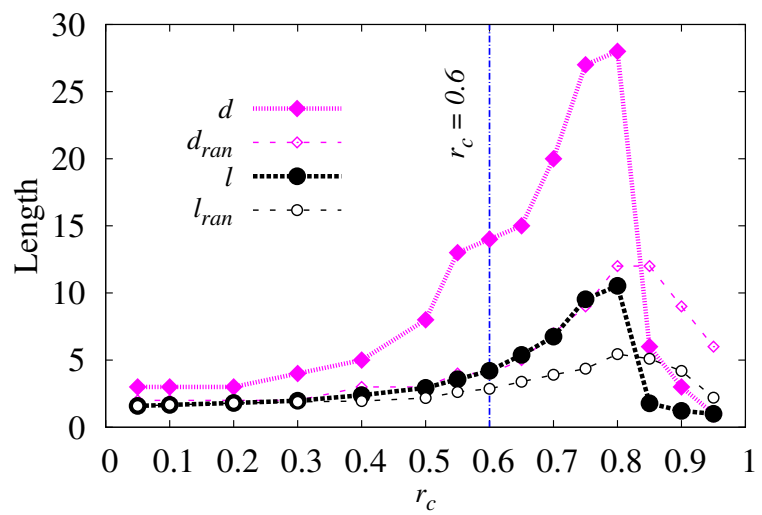

(c)

FIG. 2. (Color online) (a) The solid triangles $(\boldsymbol{\Delta})$ represent clustering coefficient $c$ for the functional network of the sandpile model, and the empty triangles $(\triangle)$ represent clustering coefficient $c_{\text {ran }}$ of the equivalent random network. (b) The cross symbols $(x)$ show the ratio of the number of vertices in the largest connected graph to the total number of the vertices in the network, $n_{c} / N$. Empty squares $(\square)$ show the density of the links $\rho_{l}$. (c) Solid diamonds $(\checkmark)$ represent the network diameter, $d$ and solid circles $(\bullet)$ represent the network average shortest path length (characteristic path length), $l$. Also empty symbols show the same parameters for the equivalent random network, respectively. The network parameters are calculated for the lattice size $L=64$. The lines are drawn only to guide eyes. 
dom graph, and they grow when the $r_{c}$ increases [see Fig. 2(c)]. The results also show a steep drop in $l$ and $d$ for $r_{c} \gtrsim 0.8$, because for the large values of $r_{c}$ the largest connected graph is vanished. These parameters are compared with the same parameters in the equivalent random network with the same number of nodes and edges and it can be seen that $d \gtrsim d_{\text {ran }}$ and $l \gtrsim l_{\text {ran }}$ in the range $0.5<r_{c}<0.8$. In sum in this range, clustering coefficient is large and the diameter and the average shortest path length are small, which means that we have a small-world network. Logarithmic behavior of the average shortest path length $l$ in terms of $n_{c}$, shown in Fig. 3, confirms that the functional network is smallworld (in preparing Fig. $3 r_{c}$ is tuned to keep the mean degree constant $\left.\langle k\rangle=2 n_{l} / n_{c}\right)$. Besides the above noted properties (indicators of a small-world network), it will be shown that in this range of the threshold for $r_{c}$ the node degree distribution of the network has scaling behavior. In the following we choose $r_{c}=0.6$ to calculate the characteristic parameters of the network, because for this value connected graph contains the largest fraction of vertices $\left(n_{c} / N\right)$, that gives best statistical results.

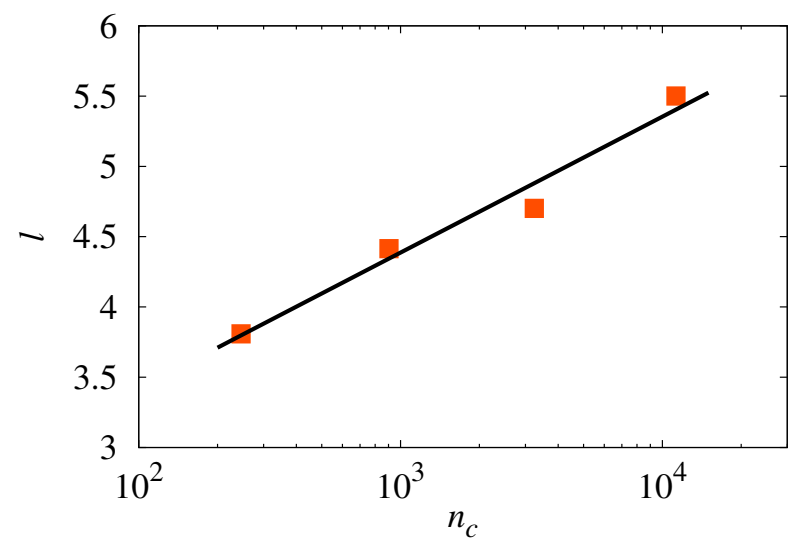

FIG. 3. (Color online) Network average shortest path length $l$ in terms of the number of vertices $n_{c}$ shows logarithmic behavior which is an indicator of the small-world network.

\section{RESULTS}

Degree distribution of the functional network of the sandpile model for three different threshold values $r_{c}$ $(0.6,0.7$, and 0.8$)$, and lattice size $L=128$ is plotted in Fig. 4. Due to the small two-point correlation in the BTW model, a fraction less than one percent of links in the complete graph appears in the degree distribution of the functional network. Scaling behavior of this graph $\left[p(k) \sim k^{-\gamma}\right]$ shows that the functional network is scalefree. Choosing $r_{c}$ in the range $[0.6,0.8]$ does not affect the scaling exponent $\gamma$ significantly, but the lattice parameters reported in the table I vary when $r_{c}$ changes, as was shown in the Fig. 2. We also derived the functional network considering anti-correlated nodes in BTW sandpile model $\left(r<r_{c}=-0.6\right)$. Consistent with the reported data for Ising model in the critical temperature [35], the resultant network is a random network with $c \sim 0.0$, $l \sim 4.4$, and $d \sim 13$, and scaling behavior of degree distribution is vanished.

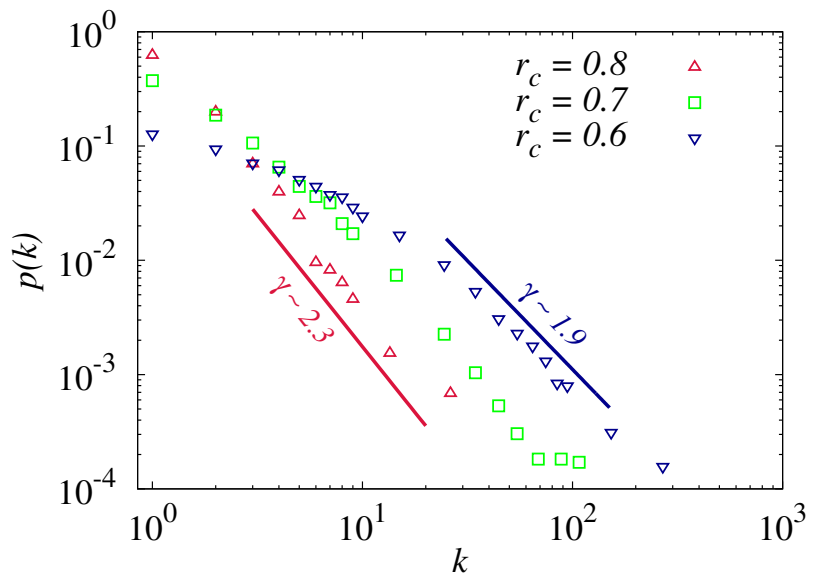

FIG. 4. (Color online) Degree distribution of the functional network of the sandpile model for three different threshold values $r_{c}$. Straight lines with a slope of 1.9 and 2.3 in the plot are drawn to guide eyes. Simulation is run for the sandpile model with lattice size $L=128$.

Fitting the three plots in Fig. 4 with regression over 0.98 shows power law behavior with exponent $\gamma=2.1 \pm$ 0.2 , which has a good agreement with the exponent of the degree distribution of the functional network of the brain [27]. Figure 4 also shows that, this model behaves similar to Ising model in two dimensions at the critical point (Fig. 5). Ising model is described by the Hamiltonian [52],

$$
H\left(\left\{\sigma_{i}\right\}\right)=-J \sum_{[i, j]} \sigma_{i} \sigma_{j}
$$

where $\sigma_{i}$ and $J$ show $i$ th spin and interaction strength, respectively, and the notation $[\cdots]$ indicates summation taken over all nearest neighbor sites.

As was reported by Fraiman et al. degree distribution of the functional network of Ising model shows scaling behavior, which indicates a scale-free network [35]. Someone may perform a power-law fitting in Fig. 5 for $r_{c}=0.7$ and 0.8 and estimate its exponent $\sim 2$. These results were repeated to ensure the validity of our statistical calculations in comparison with the corresponding results for the functional network of Ising model [35]. In addition we have used logarithmic bin in calculations to improve accuracy at the tail of distribution function (Figs. 4 and 5). Logarithmic binning method controls fluctuations and extends scaling behavior of distribution function by one order of magnitude [53, 54]. 


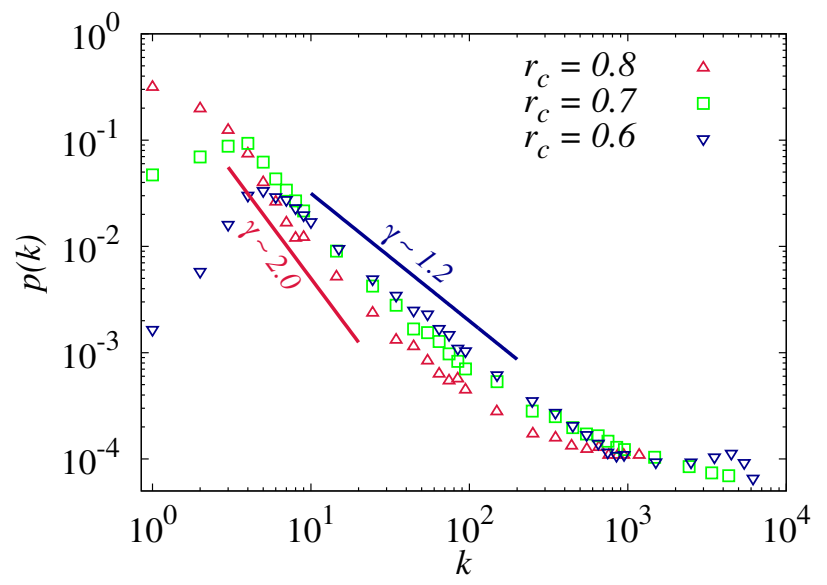

FIG. 5. (Color online) Degree distribution of the functional network of Ising model at the critical temperature for three different threshold values $r_{c}$. Straight lines with a slope of 1.2 and 2.0 in the plot are drawn to guide eyes. Simulation is run for the Ising model with lattice size $L=128$.

We have also calculated the Pearson degree-degree correlation coefficient [55],

$$
r_{\mathrm{d}-\mathrm{d}}=\frac{n_{l}^{-1} \sum_{i} j_{i} k_{i}-\left[n_{l}^{-1} \sum_{i} \frac{1}{2}\left(j_{i}+k_{i}\right)\right]^{2}}{n_{l}^{-1} \sum_{i} \frac{1}{2}\left(j_{i}^{2}+k_{i}^{2}\right)-\left[n_{l}^{-1} \sum_{i} \frac{1}{2}\left(j_{i}+k_{i}\right)\right]^{2}}
$$

where $j_{i}$ and $k_{i}$ are the degrees of the vertices which are connected by the $i$ th edge, and $\sum_{i}$ is summation over all edges, where $n_{l}$ is number of edges in graph. $r_{\mathrm{d}-\mathrm{d}}$ lies in the range of $[-1,+1]$, where $r_{\mathrm{d}-\mathrm{d}}=+1(-1)$ shows an assortative (a disassortative) mixing of nodes in the graph. The Pearson degree-degree correlation coefficient is negative for some biological networks such as the structural neural network of the nematode C. Elegans $~ 0.163$ [55]. Our results show that the coefficient is 0.299 for the functional network of BTW sandpile model, 0.274 for the functional network of Ising model at the critical temperature, and 0.183 (0.385) at a sub-(super-) critical temperature.

Other important characteristic parameters of the network for sandpile model with $r_{c}=0.6$, and for the Ising model at subcritical, critical, and supercritical temperature for $r_{c}=0.1,0.6$, and 0.09 , are calculated by using the software Cytoscape and are reported in Table I (In both of model $L=128$ ). For comparison, the results of Fraiman et al., for the functional network of brain have been added to the Table I [35].

\section{DISCUSSION}

Functional network of BTW sandpile model shows small characteristic path length scale and large clustering coefficient, in addition to the scaling behavior (see Table I and Fig. 4). The first two properties show that we are dealing with a small-world network and the last property
TABLE I. Characteristic properties of the functional networks of the BTW sandpile model, the Ising model, and the brain. 5 th row of data has been taken from the Fraiman et al. article [35].

\begin{tabular}{|c|c|c|c|c|c|}
\hline$T$ & $r_{c}$ & $N=L \times L$ & $c$ & $l$ & $d$ \\
\hline \multicolumn{6}{|c|}{ BTW sandpile model } \\
\hline- & 0.60 & 16384 & 0.461 & 1.99 & 4 \\
\hline \multicolumn{6}{|c|}{ Ising model } \\
\hline 2 . & 0.10 & 16384 & 0.083 & 3.16 & 5 \\
\hline 2.405 & 0.60 & 16384 & 0.499 & 7.76 & 35 \\
\hline 10. & 0.09 & 16384 & 0.202 & 3.36 & 5 \\
\hline \multicolumn{6}{|c|}{ Brain } \\
\hline- & 0.60 & 26985 & 0.454 & 4.4 & 13 \\
\hline
\end{tabular}

suggests that the functional network of the sanpile model is scale-free.

Nonlinear dynamics in this model is accompanied by the incidence of self-organized critical behavior. In such situations where adding a grain of sand to the sandpile might drive all of the system by events called avalanches, one can conclude existence of sites which are hubs in the network and affect many other connected sites. Such behavior may be responsible for the facilitation of communication in the network. These hubs make large clustering coefficient similar to the functional network of the brain $[1,14]$. On the other hand analysis of the fMRI images and EEG data illustrates that the avalanche behavior is involved in the transmission of data and has the essential role to speed up the communication between different parts of the brain [23].

Dynamics of BTW model is in some sense similar to the integrate-and-fire model [43]. Both of the models can be considered as a cellular automata model. Terms of rules in micro scale (evolution rule in each cell) [i.e. Eq. 2] which are used in simulating BTW model are similar to those in the integrate-and-fire model, which is widely used in the simulation of neuronal networks [43]. Leaky integrate-and-fire model is described by

$$
\begin{array}{r}
C_{m} \dot{v}_{i}=\sum_{j \neq i} I_{i j}-\frac{v_{i}}{R_{m}}, \\
\quad \text { if } v_{i}>v_{c} \longrightarrow v_{i}=0,
\end{array}
$$

where $v_{i}$ is the membrane voltage of $i$ th neuron and $I_{i j}$ is intensity of the synaptic stimulation from the $j$ th neuron to the $i$ th neuron. $v_{c}, C_{m}$, and $R_{m}$ are membrane voltage threshold, membrane capacitance, and membrane resistance, respectively. In both the models, the pressure builds up to reach a specific threshold value, then releases to the neighbor cells [i.e. in the BTW (integrateand-fire) model each sites integrates grains (stimulation) till $h_{c}\left(v_{c}\right)$ and in toppling (firing) send grains (stimulation) to the neighboring sites]. Although here the BTW is simulated on a regular lattice instead of a complex network, which is usually used for simulating neural networks. It should be noted that the BTW sandpile model with discrete dynamics of the nodes and nearest neighbor 
interactions, is not supposed to explain all the features of the brain activity such as the exponent of spatial correlation function in the blood-oxygen-level dependent of the brain resting-state activity $\sim 0.47$ reported by Eguiluz et al. $[18,27]$, and for a more concrete comparison other parameters like scaling behavior of functional network should be explored [55-57]. Interestingly a recent study shows that when the effect of noise is considered in a continuous version of the sandpile model (the stochastic parallel Zhang model), the resulted exponents are closer to those reported for the brain activity [58].

This result may reinforces qualitative impression in the reader, that if the dendritic growth mechanism extends brain network by new synaptic connections based on the correlations in the neural dynamics, even if the initial structure of the brain network is a regular network, the final will be a scale-free and small-world network [37, 59]. However it should not be forgotten that the structural network and the function network of brain have fundamental differences [60].

\section{ACKNOWLEDGMENTS}

Authors would like to thank Sergio A. Cannas for his valuable comments on the manuscript and Abdorreza Goodarzi Nick for his helps in running SNAP.
[1] D. R. Chialvo, Nat. Phys. 6, 744 (2010).

[2] P. Dayan and L. F. Abbott, Theoretical neuroscience: Computational and Mathematical Modeling of Neural Systems, Vol. 31 (MIT press, Cambridge, Massachusetts, 2001).

[3] M. Paczuski, S. Maslov, and P. Bak, Phys. Rev. E 53, 414 (1996).

[4] B. Gutenberg and R. C. F., Seismicity of the earth and associated phenomena" (Princeton University Press, New Jersey, 1956).

[5] T. E. Harris, The theory of branching processes (Dover, New York, 1989).

[6] B. D. Malamud, G. Morein, and D. L. Turcotte, Science 281, 1840 (1998).

[7] P. Bak, C. Tang, and K. Wiesenfeld, Phys. Rev. Lett. 59, 381 (1987).

[8] J. B. Bassingthwaighte, L. S. Liebovitch, and B. J. West, Fractal physiology (Oxford University Press, New York, 1994).

[9] A.-L. Barabási and H. E. Stanley, Fractal concepts in surface growth (Cambridge University Press, United Kingdom, 1995).

[10] P. Bak, How nature works: the science of self-organized criticality (Copernicus Press, New York, 1996).

[11] J. M. Beggs and D. Plenz, J. Neurosci. 23, 11167 (2003).

[12] D. Plenz, E. Niebur, and H. G. Schuster, eds., Criticality in Neural Systems, Reviews of Nonlinear Dynamics and Complexity (Wiley, Verlag, 2014).

[13] H. H. Berra, P. Balenzuela, D. Fraiman, and D. R. Chialvo, in Encyclopedia of Life Support Systems (EOLSS), Complex Networks, Vol. 1, edited by G. Caldarelliaytexas (Eolss Publishers, Paris, France, 2010) p. 234, developed under the Auspices of the UNESCO.

[14] D. R. Chialvo, New Ideas Psychol. 26, 158 (2008).

[15] D. Plenz and T. C. Thiagarajan, Trends Neurosci. 30, 101 (2006).

[16] J. M. Palva, A. Zhigalov, J. Hirvonen, O. Korhonen, K. Linkenkaer-Hansen, and S. Palva, Proc. Natl. Acad. Sci. U.S.A. 110, 3585 (2013).

[17] C. Anteneodo and D. R. Chialvo, Chaos 19, 033123 (2009).

[18] P. Expert, R. Lambiotte, D. R. Chialvo, K. Christensen, H. J. Jensen, D. J. Sharp, and F. Turkheimer, J. R. Soc. Interface 8, 472 (2011).
[19] D. R. Chialvo, P. Balenzuela, and D. Fraiman, AIP Conf. Proc. 1028, 28 (2008).

[20] D. V. D. Ville, J. Britz, and C. M. Michel, Proc. Natl. Acad. Sci. U.S.A. 107, 18179 (2010).

[21] C. Bédard, H. Kröger, and A. Destexhe, Phys. Rev. Lett. 97, 118102 (2006).

[22] G. Buzsáki, Rhythms of the Brain (Oxford University Press, New York, 2006).

[23] W. L. Shew, H. Yang, T. Petermann, R. Roy, and D. Plenz, J. Neurosci. 92, 59551 (2009).

[24] T. Tanaka, T. Kaneko, and T. Aoyagi, Neural. Comput. 21, 1038 (2009).

[25] O. Kinouchi and M. Copelli, Nat. Phys. 2, 348 (2006).

[26] J. S. Damoiseaux, S. A. R. B. Rombouts, F. Barkhof, P. Scheltens, C. J. Stam, S. M. Smith, and C. F. Beckmann, Proc. Natl. Acad. Sci. U.S.A. 103, 13848 (2006).

[27] V. M. Eguíluz, D. R. Chialvo, G. A. Cecchi, M. Baliki, and A. V. Apkarian, Phys. Rev. Lett. 94, 018102 (2005).

[28] X. Liu, B. D. Ward, J. R. Binder, S.-J. Li, and A. G. Hudetz, PLoS ONE 9, e92182 (2014).

[29] G. Grega Repovs, J. G. Csernansky, and D. M. Barch, Biol. Psychiatry 69, 967 (2011).

[30] Y. He, Z. Chen, and A. Evans, J. Neurosci. 28, 4756 (2008).

[31] I. Osorio, M. G. Frei, D. Sornette, J. Milton, and Y.-C. Lai, Phys. Rev. E 82, 021919 (2010).

[32] C. J. Honey, O. Sporns, L. Cammoun, X. Gigandet, J. P. Thiran, R. Meuli, and P. Hagmann, Proc. Natl. Acad. Sci. U.S.A. 106, 2035 (2009).

[33] J. O'Reilly, P. L. Croxson, S. Jbabdi, J. Sallet, M. P. Noonan, R. B. Mars, P. G. F. Browning, C. R. E. Wilson, A. S. Mitchell, K. L. Miller, M. F. S. Rushworth, and M. G. Baxter, Proc. Natl. Acad. Sci. U.S.A. 110, 13982 (2013).

[34] J. Johnston, S. N. Vaishnavi, M. D. Smyth, D. Zhang, B. J. He, J. M. Zempel, J. S. Shimony, A. Z. Snyder, and M. E. Raichle, J. Neurosci 28, 6453 (2008).

[35] D. Fraiman, P. Balenzuela, J. Foss, and D. R. Chialvo, Phys. Rev. E 79, 061922 (2009).

[36] E. Helfand and J. S. Langer, Phys. Rev. 160, 437 (1967).

[37] D. J. Watts and S. H. Strogatz, Nature 393, 440 (1998).

[38] A.-L. Barabási and E. Bonabeau, Sci. Am. 288, 60 (2003).

[39] P. Bak, C. Tang, and K. Wiesenfeld, Phys. Rev. A 38, 
364 (1988).

[40] J. M. Carlson and G. H. Swindle, Proc. Natl. Acad. Sci. U.S.A. 92, 6712 (1995).

[41] S. Dodel, J. M. Herrmann, and T. Geisel, Neurocomputing 44, 1065 (2002).

[42] S. Lübeck, N. Rajewsky, and D. E. Wolf, Eur. Phys. J. B 13, 715 (2000).

[43] L. F. Abbott, Brain Res. Bull. 50, 303 (1999).

[44] D. Dhar, "Studying self-organized criticality with exactly solved models," (1999), arXiv:cond-mat/9909009 [condmat.stat-mech].

[45] J. Feder, Fractals (Plenum Press, New York, 1988).

[46] W. H. Press, Comments Astrophys. 7, 103 (1978).

[47] M. B. Weissman, Rev. Mod. Phys. 60, 537 (1988).

[48] D. Dhar, Physica A 369, 29 (2006).

[49] What we call here the height of the site, is actually the difference of the height of the site with the height of its neighbors (slope of the site).

[50] P. Shannon, A. Markiel, O. Ozier, N. S. Baliga, J. T. Wang, D. Ramage, N. Amin, B. Schwikowski, and
T. Ideker, Genome Res. 13, 2498 (2003).

[51] J. Leskovec and R. Sosič, "SNAP: A general purpose network analysis and graph mining library in $\mathrm{C}++, "$ http://snap. stanford.edu/snap (2014).

[52] E. Ising, Z. Physik 31, 253 (1925).

[53] D. W. Sims, D. Righton, and J. W. Pitchford, J. Anim. Ecol. 76, 222 (2007).

[54] E. P. White, B. J. Enquist, and G. J. L., Ecology 89, 905 (2008).

[55] M. E. J. Newman, Phys. Rev. Lett. 89, 208701 (2002).

[56] C. Song, S. Havlin, and H. A. Makse, Nature 433, 392 (2005).

[57] S. D. Reis, Y. Hu, A. Babino, J. S. Andrade Jr, S. Canals, M. Sigman, and H. A. Makse, Nat. Phys., 762 (2014).

[58] S. A. Moosavi and A. Montakhab, Phys. Rev. E 89, 052139 (2014).

[59] G. Caldarelli, A. Capocci, P. De Los Rios, and M. A. Muñoz, Phys. Rev. Lett. 89, 258702 (2002).

[60] O. Sporns, D. R. Chialvo, M. Kaiser, and C. C. Hilgetag, Trends Cogn. Sci. 8, 418 (2004). 